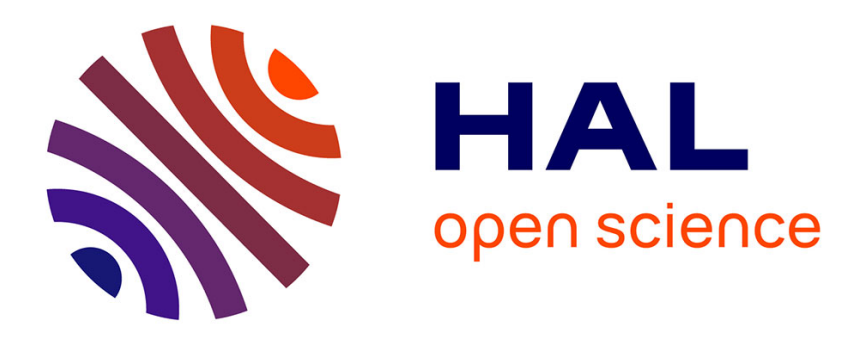

\title{
L'économie du livre saint dans le Maroc contemporain
} Anouk Cohen

\section{To cite this version:}

Anouk Cohen. L'économie du livre saint dans le Maroc contemporain. Les Cahiers du CAP - Créations Arts Patrimoine, 2015. hal-01631945

\section{HAL Id: hal-01631945 https://hal.parisnanterre.fr/hal-01631945}

Submitted on 18 Dec 2020

HAL is a multi-disciplinary open access archive for the deposit and dissemination of scientific research documents, whether they are published or not. The documents may come from teaching and research institutions in France or abroad, or from public or private research centers.
L'archive ouverte pluridisciplinaire HAL, est destinée au dépôt et à la diffusion de documents scientifiques de niveau recherche, publiés ou non, émanant des établissements d'enseignement et de recherche français ou étrangers, des laboratoires publics ou privés. 


\section{Anouk Cohen L'économie du Livre saint
dans le Maroc contemporain}

À Casablanca, dans le quartier des Habous ${ }^{1}$ qui concentre plus d'une soixantaine de maisons d'édition et de distribution en gros et au détail, le Coran est qualifié de «best-seller» par les éditeurs. Vendu par milliers tous les mois, il fait l'objet d'un engouement grandissant ces dernières années, notamment dû aux récents progrès de l'alphabétisation. Ce «boom» du Livre saint a encouragé de nombreux libraires des Habous à lui accorder une place privilégiée dans leurs boutiques. Il est fréquent de le voir exposé dans les vitrines, en position ouverte, installé sur un lutrin en bois le maintenant légèrement en hauteur. Un procédé visant selon les libraires à attirer la clientèle. De la vente du Coran dépend même la vente d'autres livres, religieux ou littéraires, poursuit le libraire : «Si je n'ai pas le modèle de Coran qu'il recherche, le client ira l'acheter avec tous les autres livres dont il a besoin dans une autre librairie, à côté ${ }^{2}$.» Cette remarque est confirmée par de nombreux commerçants du quartier qui déclarent importer un nombre de plus en plus important d'exemplaires de Coran³. Certains libraires ont même entrepris de produire leur propre modèle de Livre saint afin de mieux répondre aux attentes de leurs clients et optimiser leurs ventes.

Ces observations amènent à formuler deux interrogations principales : de quelle manière les éditeurs/distributeurs du quartier des Habous parviennent-ils à faire du Coran un «best-seller»? Quelles sont les implications économiques, religieuses et politiques de la transformation de ce livre au statut si particulier en une marchandise? Autant d’interrogations placées au cœur de la réflexion axée sur l'étude du marché coranique et son fonctionnement à un moment où il connaît une profonde

1 Érigé entre les années 1920 et les années 1940, le quartier des Habous dessiné par Albert Laprade en 1917 a été construit à l'époque du Protectorat, dans le cadre de la politique urbaine du général Lyautey afin de séparer les lieux de résidence européens des lieux de résidence marocains. Les biens immobiliers qu'il renferme appartiennent à l'État qui les affecte à des œuvres de bienfaisance (construction et équipement de médersas - écoles islamiques -, versement des pensions aux étudiants et des salaires des enseignants, etc.). C'est pourquoi il porte le nom de «Habous», ce terme signifiant en arabe : «l'acte de fondation d'une institution charitable, d'où l'institution elle-même» (Peters, 2005 [1960]). En outre, le radical WQF comme HBS d'où vient habous, signifie «arrêter», «immobiliser».

2 Entretien du 10 octobre 2012 à Casablanca.

3 Malheureusement, les statistiques manquent dans ce domaine. Le ministère de la Communication et de l'Information chiffre les importations de livres divers sans distinguer les ouvrages. 
transformation, liée à la création en 2010 d'une édition nationale du Coran. Cette disposition vise à contrôler et à encadrer la circulation du texte coranique dans les mosquées du royaume : un enjeu crucial au Maroc où l'autorité du roi, descendant du Prophète et «Commandeur des croyants », est en partie fondée sur le contrôle du rapport de la société à la religion (Tozy, 1999). Pour mener à bien cette étude, je me suis prioritairement intéressée à deux types d'acteurs : les producteurs de Coran et l'État4.

\section{Coran : «best-seller » des Habous}

\section{Controverses religieuses}

Depuis le Moyen Âge, la question de la licéité de l'édition et de la vente du Livre saint est au cœur de disputes religieuses. Afin de mieux cerner les débats qui divisent les différents courants musulmans, il est utile de rappeler la conception particulière de la révélation dans l’islam.

À l'inverse de la Bible, le Coran ne se revendique pas tant comme une Écriture «sainte» que comme Écriture «divine» (Prémare, 2004, p. 27). «Cette revendication s'exprime, au niveau théologique, par l'affirmation du tanzîl, la "descente”, conçue comme mode opératoire de la révélation du livre»(ibid.). Le Coran - que l'arabe désigne par mushaf (volume) pour ne pas confondre la révélation (qur'ân) avec son support (Burton, 1993 [1966]) - est, aux yeux des musulmans, le livre de Dieu (kitâb allâh). Cette représentation du Livre saint a eu un impact important sur sa production et sa diffusion. L'adoption tardive de l'imprimerie dans le monde islamique, près de quatre siècles après son avènement en milieu chrétien, en est la parfaite illustration 5 .

Les premières éditions imprimées du texte original arabe du Coran voient le jour en Europe au XVII ${ }^{\text {e }}$ siècle : éditions partielles à partir de 1617 en Hollande, édition intégrale à Hambourg en 1694, à Padoue en 1698 et à Saint-Pétersbourg en 1787 (Albin, 2005). À l'exception de celle-ci réalisée sous le patronage de Catherine II de Russie

4 Cette étude s'appuie sur une enquête menée au sein de trois maisons d'édition et de distribution du quar-

tier des Habous au cours de différents séjours entre 2010 et 2013. La méthode ethnographique employée a consisté à travailler en tant que libraire dans ces maisons durant plusieurs semaines. En outre, ma présence quotidienne sur ces lieux d'observation m’a permis de réaliser des entretiens avec plus d'une quarantaine de professionnels et une trentaine de clients. Essentiellement axé sur l'édition coranique et sa récente transformation, cet article ne porte pas attention aux manières dont le Livre saint est vendu au détail et reçu par les usagers. Pour plus d'informations à ce sujet, voir Cohen (2010). Je tiens à remercier Roger Chartier et Constant Hamès pour leurs commentaires sur la version provisoire du texte.

5 Rappelons cependant que l'introduction de l'imprimerie a également fait l'objet de disputes religieuses chez les chrétiens dont l'un des principaux enjeux était la forme prise par le Livre. Sur ce point voir Gilmont (1997) et McKenzie (1991). Pour plus d'informations sur l'histoire du livre arabe et l'impact de l'imprimé dans le monde islamique, voir notamment Pedersen (1984), Balagna (1984), Atiyeh (1995), Gdoura (1985), Robinson (1991, 2003), Cole (2002), Hirschkind (2005), Berthier, Héricher, Zali (2005), Mermier (2005). 
avec l'aide du mollah 'Uthman Ismâ'îl, ces éditions sont largement destinées à un public chrétien, savant ou polémiste, qui n’a jusque-là pas accès au texte. Aussi, ne reçoivent-elles aucun écho dans le monde musulman (Hamès, 2013). C'est en 1923 au Caire à Al-Azhar, principale université d'étude islamique, qu'apparaît sous l'égide du roi Fouad I ${ }^{\text {er }}$, le premier Coran imprimé en caractères mobiles. Ce Coran dit «Coran du Caire » est rapidement devenu l'équivalent d'une édition «officielle », largement diffusée à travers le monde musulman (Azaiez6, 2013, p. 15). Aujourd'hui, elle sert de modèle à la grande majorité des masahîf (pluriel de mushaf) imprimés dans la région.

Les difficultés techniques, la place économique importante occupée par la corporation des copistes et le prestige de l'écriture manuscrite sont autant de freins à l'introduction de l'imprimerie dans le monde islamique. Mais la résistance à imprimer dans la langue du Coran, en particulier le texte révélé, tient surtout à des raisons religieuses, culturelles et politiques : l'élite des oulémas, docteurs de la Loi, craint que le Livre ne soit altéré par sa reproduction technique et que la standardisation de l'imprimé ne bouleverse le système de transmission orale du savoir au fondement de son autorité. En outre, les musulmans sont horrifiés par des premières éditions du Coran réalisées par les Européens. De pauvre qualité, beaucoup d'entre elles présentent des erreurs, comme de nombreux livres imprimés en Europe à l'époque de la Renaissance (Eisenstein, 1979) ${ }^{7}$.

Sous la pression des oulémas, les sultans ottomans Bayezid II et Selim I ${ }^{\text {er }}$ décrètent en 1485 et en 1515 l'interdiction de l'imprimerie ${ }^{8}$. Sa levée partielle au XV $\mathrm{XV}^{\mathrm{e}}$ siècle exclut l'édition des livres religieux musulmans, qui n'apparaît en Turquie et dans ses provinces qu'à la fin du XIX ${ }^{\mathrm{e}}$ siècle. Parallèlement à ces tentatives, se met en place une autre technique apparue en Allemagne en 1796 : la lithographie, qui connaît un vif succès dans le monde islamique (Berthier, Héricher, Zali, 2005). En reproduisant le tracé effectué à l'encre ou au crayon sur une pierre calcaire ou une plaque métallique, cette technique permet de reproduire l'écriture manuelle. Ainsi, la lithographie ne bouscule pas les habitudes du fidèle qui n'est familiarisé qu'avec l'écriture manuscrite adoptée pour la planchette coranique, principal outil d'apprentissage des versets dans le kuttâb (école coranique). L'enfant, une fois qu'il maîtrise l'alphabet, reçoit une tablette en bois sur laquelle il doit reproduire telle quelle l'écriture des versets à partir d'un

6 Cet auteur reprend l'expression formulée par Bergsträsser (1932).

7 Ce souci d'exactitude s'exprime par le terme i'jâz, utilisé dès la seconde moitié du Ix siècle pour désigner le caractère inimitable et unique du Coran, aussi bien dans son contenu que dans sa forme (Baqillani, 1964).

8 Notons que la propagande religieuse et les rivalités confessionnelles ont joué un rôle central dans la diffusion de l'imprimerie à caractères mobiles dans le monde arabe. Dès 1578, le Collège maronite de Rome invite de jeunes clercs de la communauté maronite libanaise à fréquenter les collèges romains afin qu'ils y étudient le nouvel art. À son retour de Rome, l'un d'entre eux rapporte une imprimerie qu'il installe au couvent Saint-Antoine de Quzhaya où est imprimé en 1610 le premier livre au Liban : un psautier. Ce n'est qu'un siècle plus tard, en 1704, qu'une imprimerie arabe est établie à Alep (Nasrallah, 1951). 
calque confectionné par son maître. Sa planche en bois maintenue à la verticale sur ses genoux, l'enfant répète le texte tout en le copiant (Fortier, 2003). Mémorisation, récitation et mise à l'écrit font partie intégrante de cet apprentissage que les musulmans privilégient de façon quai exclusive jusqu'à la fin du XIX siècle (Graham, 1987; Messick 1996; Touati, 200o). À son apparition dans le monde islamique, la reproduction mécanique frappe au cœur ce système de transmission qui repose sur la relation directe du maître et de ses élèves (Robinson, 1991, 2003).

Au Maroc, où ce mode d'apprentissage - encore largement employé dans les écoles coraniques qui accueillent plus de $88 \%$ des enfants scolarisés entre 4 et 7 ans (Bouzoubaa, Benghabrit-Remaoun, 2004) - est particulièrement ancré (Eickelman, 1992; Geertz, 1986), la résistance à imprimer se fait plus forte qu'ailleurs dans la région. L'imprimerie n'y apparaît qu'en 1865, c'est-à-dire plus de cent ans après l'établissement, on l'a dit, de la première unité arabe à Alep (1704). En outre, la typographie est adoptée après la lithographie. D’ailleurs, la première édition imprimée au Maroc du Coran est une copie lithographiée réalisée en 1879, plusieurs décennies avant celle à caractères mobiles (Albin, 2005) ${ }^{9}$. Il est probable que cet écart tienne à la calligraphie ayant servi de modèle à la réalisation des caractères typographiques arabes : le naskhî, différente de celle employée dans les écoles coraniques marocaines, le maghribî. En effet, le passage de la copie manuscrite à la typographie s'accompagne d'un changement d'écriture qui peut avoir une incidence sur l'apprentissage du Coran dans la mesure où l'orthographe - entendue dans son acception pleine : la graphie correcte suivant les standards d'usage, la prononciation et la grammaire des mots - on l'a dit, y occupe une place centrale (Rezvan, 2005).

L'opposition forte à l'imprimerie au Maroc tient principalement au conservatisme des oulémas disciples du juriste traditionaliste Malik b. Anas (d. 179-796) (Laroui, 1993), fondateur de l'une des quatre écoles juridiques sunnites : le malékisme, toujours en vigueur dans le pays ${ }^{10}$. Elle est surtout liée à l'attitude prudente du sultan face à l'élite religieuse qui constitue une force politique non négligeable avant le Protectorat (1912-1956). En témoigne le dahîr (décret royal) de 1897 qui accorde au

9 Rappelons ici la révérence particulière des populations musulmanes d’Afrique du Nord pour le Coran, qui se manifeste notamment à travers l'adoption tardive non seulement de l'imprimerie mais aussi du papier (Bloom, 2001). Les caractéristiques d’anciens manuscrits du Coran, sobrement décorés, sont une autre expression de ce conservatisme. Le format carré qui permettait de distinguer le Coran d’autres livres profanes, de sorte qu'il fasse l'objet d'un comportement approprié, a d'ailleurs perduré jusqu'au xve siècle dans la région (Déroche, 2005). Enfin, les oulémas traditionalistes ont retardé l'introduction des manuscrits dans les mosquées car elle portait préjudice à la primauté qui devait normalement être accordée à la mémorisation du Coran et à l'importance pour le fidèle de le porter dans son cœur.

10 Le malékisme est l'une des quatre écoles juridiques de l'islam sunnite. Décrite comme traditionaliste, cette école est majoritaire en Afrique du Nord et en Afrique de l’Ouest. On la retrouve également en Égypte, au Soudan et dans certains pays du Golfe Persique (Laroui, 1993). 
cadi (juge musulman) de Fès, capitale religieuse, le droit de superviser le contenu des livres imprimés (Laroui, op. cit.). Aussi, il est intéressant que les quelque cinq cents titres imprimés entre 1865 et la date de l'avènement du Protectorat sont ou bien des manuels en usage depuis plus de deux siècles (ouvrages de droit islamique fiqh et de grammaire) à l'université-mosquée de la Qarawiyîn, principal centre de diffusion du savoir islamique, ou bien des textes hagiographiques traditionnels (Ayach, 1964). Ainsi, lorsque l'imprimerie fait son entrée au Maroc, elle est prioritairement mise au service des traditions par les élites religieuses et politiques, contrairement à l'Égypte et au Liban par exemple où elle joue un rôle «modernisateur» (Sghir Janjar, 2006) ${ }^{11}$.

Ce n'est qu'à partir de 1956, à la fin du Protectorat, que les besoins de l'indépendance ont favorisé le développement de l'édition et de l'imprimerie marocaines (Unesco, 1987). Jusqu'à une période récente, le livre scolaire domine le secteur et l'édition culturelle indépendante se résume à quelques actions ponctuelles. La majorité des romans, essais, ouvrages pratiques, livres islamiques, etc. sont importés de France et des pays du Moyen-Orient (en particulier l'Égypte et le Liban). Ce n'est pas avant 1985 qu'un marché éditorial commence à se constituer. L'augmentation du volume de la production éditoriale et l'apparition de jeunes maisons d'édition proposant, dans leur ensemble, des nouveaux titres culturels sont les principales manifestations de la transformation du secteur. Depuis l'année 200o, le Maroc commence à publier en un an l'équivalent de ce qu'il a publié durant près d'un siècle (1865-1955) (Sghir Janjar, op. cit.). Comme avant le Protectorat, l'islam est au cœur d'une bonne part des projets éditoriaux contemporains (ibid.). Et, chose nouvelle et remarquable, le Coran suscite de plus en plus l'intérêt des éditeurs. ll s'agit à présent de comprendre les implications économiques, religieuses et politiques de la mise en place d'un nouveau marché éditorial coranique dans un pays comme le Maroc où les élites, on l'a dit, ont toujours fait preuve d'un conservatisme particulier. À cette fin, il me faut d'abord décrire comment ce nouveau marché coranique est en train d'émerger dans le quartier des Habous.

\section{Nouveaux publics et usages du Livre saint : la naissance d'un marché}

Contrairement au Caire où une grande variété de modèles de Coran est vendue en nombre important depuis le milieu des années 1980 (Starrett, 1995, 1998), à Rabat et à Casablanca le «boom» du Livre saint est relativement récent. Il est lié aux changements

11 Dans ces pays, en effet, l'imprimerie a été un outil essentiel de la Nahda, la «Renaissance» arabe au XIX ${ }^{\mathrm{e}}$ siècle. Ce mouvement a impulsé des tentatives de réforme de la langue arabe (Haeri, 2003). 
culturels (progrès de l'alphabétisation ${ }^{12}$, hausse du taux de scolarisation, apparition d'Internet en 1996) et sociaux (réforme du code de la famille, extension des droits de la femme ${ }^{13}$ ) permis par une libéralisation politique plus forte depuis le milieu des années 1990 et le début du règne de Mohammed VI (1999).

La hausse du pouvoir d'achat est un autre facteur essentiel du développement du marché éditorial coranique. De 1700 dirham (dh) en 2000 (Vermeren, 2001), le salaire minimum est passé à 2333 dh en $2013^{14}$. Une information cruciale dans un pays où la vente des livres est sensible à la moindre modification du pouvoir d'achat (El Yazami, 2007). D’autant que le prix du livre du Coran, compris entre 3 dh (presque o,20 centimes d'euro) et 500 ooo dh (50 ooo euros) pour les modèles les plus sophistiqués, est de plus en plus abordable. À titre d'exemple, le livre du Coran de moyen format est vendu $20 \mathrm{dh}$ environ, un prix qui casse radicalement le marché du livre local, un ouvrage produit au Maroc coûtant environ $70 \mathrm{dh}$, soit trois fois plus qu'un exemplaire de Coran, alors qu'il présente une qualité esthétique moindre.

Ces changements sont en train d'étendre l'accès du Livre saint à d'autres catégories de la population, au-delà de la seule élite intellectuelle et économique, en particulier les femmes, les classes populaires et les jeunes scolarisés (de 12 à 25 ans). Comme ce fut le cas autrefois avec la Bible en France après que le pays eut connu une période de scolarisation massive ${ }^{15}$, le livre que ces nouveaux lecteurs ${ }^{16}$ s'approprient en premier est le livre religieux : en l'occurrence le Coran qui constitue l'héritage référentiel, celui qu'une grande partie des Marocains souhaitent connaître en priorité ${ }^{17}$. En outre, les études menées par Bourqia, El Ayadi, El Harras, Rachik en 200o, complétées en 2007

12 Le taux d’analphabétisme de la population âgée de plus de 10 ans a été ramené de $43 \%$ en 2004 à près de $28 \%$ en 2013. À Rabat et à Casablanca, les capitales économique et administrative du pays, plus de $70 \%$ de la population sait lire et écrire. Voir «Baisse du taux d'analphabétisme au Maroc à 28\%», La Vie éco (http:// www.lavieeco.com/news/actualites/baisse-du-taux-d-analphabetisme-au-maroc-a-28--26483.html, consulté le 6 juin 2014) et le rapport officiel 50 ans de développement humain au Maroc (2006).

13 Les femmes sont les principales bénéficiaires des programmes d’alphabétisation mis en place par le royaume ces dix dernières années. En outre, depuis la réforme du Code de la famille en 2000, elles sont de plus en plus nombreuses à être scolarisées et à intégrer l'université. Voir «Baisse du taux d'analphabétisme au Maroc à $28 \% \gg$, La Vie éco, site cité.

14 Voir http://www.mariage-franco-marocain.net/article-smig-au-maroc-montant-du-smig-marocain-2013-smichoraire-119287632.html et http://www.infomaroc.net/economie/23527-le-revenu-mensuel-moyen-par-menagemarocain-seleve-a-pres-de-5300-dirhams-hcp.html

15 À ce sujet, se reporter aux travaux de Chartier et Martin (1989-1990 [1986-1990]), Laplanche (1994) et Lassave (2011).

16 L'expression «nouveaux lecteurs» est utilisée dans un autre contexte par Jean Hébrard. Elle désigne : «Ceux pour qui l'apprentissage de la lecture est une véritable acculturation, un arrachement à des générations d'analphabétisme qui suppose un effort de tous les instants, une volonté exacerbée, des stratégies complexes.» (Hébrard, 1990, p. 590)

17 Une observation corroborée par une enquête de l'Unesco selon laquelle «le besoin de lire le Coran est l'un des principaux facteurs avancés par les bénéficiaires pour justifier leur participation à des programmes d’alphabétisation» (2005). 
de celles d'El Ayadi, Rachik, Tozy, montrent que le Coran-livre occupe une place grandissante dans l'apprentissage religieux des jeunes générations. Celles-ci cherchent à accéder directement au savoir islamique en dehors du système de tradition personnalisée des pères, professeurs d'école et imams (Tozy, 2009, p. 65). «Comme le rappelait Berque dans le Maghreb entre les deux guerres, le hizf (mémorisation) cède la place à la moutala'a (lecture avec prise de notes) » (ibid.). Il est intéressant qu'une part importante des clients des Habous cherchent à acquérir leur «propre» exemplaire de Coran (Cohen, 2010). Ils sont également nombreux à offrir le Livre saint et à le recevoir en cadeau. Cette pratique récente est de plus en plus courante à Rabat et à Casablanca (ibid.). Elle entraîne à son tour une augmentation des ventes des libraires.

Ce «boom» du Livre saint est allé de pair avec l'apparition de nouveaux usages, notamment l'apprentissage «par cœur» du Coran aussi bien par les hommes que par les femmes, les adultes et les jeunes, issus des milieux lettrés ou non (Cohen, 2012). Depuis les débuts de l'islam, la mémorisation du texte - de quelques versets fondamentaux comme la Fatiha (la liminaire) à son intégralité - constitue un trait constant de la dévotion que les fidèles vouent à la révélation (Déroche, 2005). La plus grande circulation du texte coranique est en train de donner un nouveau souffle à cette pratique, redessinant ainsi les contours d'une catégorie professionnelle ancestrale dans les sociétés musulmanes : les qâri' (du verbe qarâ'a qui désigne à la fois l'acte de réciter tout ou partie du Coran et la façon de le lire/réciter). Ces «récitateurs » connaissent par cœur le Coran. Au Maroc, comme ailleurs, tant dans les campagnes que dans les villes, les croyants engagent un de ces spécialistes pour réciter des extraits du Coran après un enterrement, une naissance ou bien au cours d'un mariage, une circoncision, etc. (Déroche, 2008 [2005]). Si cette profession a toujours représenté un signe de promotion sociale dans le royaume (Eickelman, op. cit.), aujourd'hui, elle constitue davantage un signe de promotion économique. De plus en plus, l’idée que par le seul apprentissage du Coran «on peut s'en sortir » - c'est-à-dire recevoir une rémunération en échange d'un travail fourni - se répand dans les milieux populaires. C'est pourquoi un nombre croissant d'individus, notamment de jeunes diplômés chômeurs, cherche à mémoriser le Coran dans son intégralité. En 2004, le taux de chômage s'élève à 10,8\% de la population active au Maroc et touche près de $27 \%$ des jeunes diplômés universitaires. Les diplômés dont le cursus est entièrement arabophone et strictement littéraire sont les premiers à être touchés (Emperador, 2007). Ce sont également ceux qui ont les meilleures compétences pour devenir qâri'.

Dans ce but, ces qâri' autodidactes, suivis d'autres fidèles, achètent plusieurs modèles de Coran - trois en moyenne de différents formats - de manière à pouvoir le lire n'importe où et en toutes circonstances. Le "grand Coran», plus lisible, est principalement utilisé à la maison où il est généralement posé sur un lutrin ; le moyen, le mieux vendu, est utilisé aussi bien dans les habitations que sur le lieu de travail et dans les transports en commun. Mais c'est le petit format - en particulier celui recouvert d'un 
étui en skaï à fermeture Éclair - qui est le plus répandu dans les bus, les trains et les taxis. Ce modèle permet aux fidèles de transporter le texte partout sans en abîmer les pages. Enfin, les livres de Coran miniatures auxquels sont associés des usages principalement talismaniques (Hamès, 2007), font également partie des meilleures ventes des libraires du quartier des Habous. Leur best-seller reste cependant le Coran de moyen format acheté par centaines chaque semaine par un ou plusieurs fidèles souhaitant en faire des donations pieuses (waqf) aux mosquées. Selon un éditeur/distributeur du quartier, «ces achats représentent le plus gros chiffre d'affaires ${ }^{18} »$.

Ainsi, la nouvelle conjoncture sociale, culturelle et économique explique le succès commercial important du Coran pour ces commerçants. Ceux-ci n'hésitent d'ailleurs pas à le vendre aux côtés d'autres objets cultuels - comme le tapis de prière, les cassettes de récitation coraniques ou encore les lutrins - dont le Livre saint, disent-ils, dynamise les ventes. Mis ensemble, ces objets forment le «kit religieux» du fidèle, celui que chaque Marocain se doit d'avoir ${ }^{19}$. Le marché coranique local n'a jamais été aussi florissant que ces dernières année. Loin d'être seulement un livre sacré, le Coran est une marchandise de plus en plus placée au cœur de rivalités commerciales au quartier des Habous.

\section{Les «bisnessmen » du Coran}

\section{S'improviser éditeur de livres saints}

Les masahîf vendus aux Habous proviennent majoritairement du Caire, de Beyrouth et de Ryad où ils sont produits par centaines de milliers d'exemplaires, ce qui réduit considérablement les coûts à l'unité (Mermier, 2005). En outre, l'exonération de droits de douanes sur l'importation de livres culturels (qui inclut les livres religieux et exclut les livres scolaires) diminue encore les frais initiaux. C'est pourquoi, le Maroc représente un marché attractif pour les éditeurs moyen-orientaux, qui sont nombreux chaque mois à se rendre à Casablanca pour vendre leur catalogue aux éditeurs-distributeurs des Habous. À l'intérieur des boutiques, les éditeurs libanais,

18 Entretien du 10 octobre 2012 à Casablanca.

19 Dans le cadre de cette réflexion, il serait intéressant d’analyser dans quelle mesure la baisse du coût du livre du Coran encourage les fidèles à reporter une partie de leurs dépenses sur ces produits dérivés du Livre saint. Cette étude pourrait être complétée par une enquête sur les manières dont les éditeurs des Habous, soucieux de répondre aux besoins d'un public grandissant et de plus en plus varié, cherchent à concevoir différents modèles de Coran. Et comment, dans ce but, les baisses de coûts de production du texte coranique leur permettent de reporter une partie de leurs investissements sur le support, c'est-à-dire l'objet-livre luimême enrichi de nouveaux matériaux (tels que les pierres précieuses ou la silicone) et accessoires (comme le stylo électronique audio). 
égyptiens, syriens et marocains négocient le prix des modèles qu'ils souhaitent vendre ou acquérir jusqu'à obtenir une réduction qui les satisfasse. Le but des commerçants du quartier des Habous qui proposent à peu de chose près les mêmes types d'ouvrages (livres scolaires, ouvrages islamiques, Coran, dictionnaires, romans arabes) est de les vendre au meilleur prix. Au Maroc, comme ailleurs dans le monde arabe, le prix du livre n'est pas fixe. La pratique veut qu'un livre fabriqué au Liban ou ailleurs se voie attribuer un prix de vente différent dans le pays où il est importé, qui tienne compte du niveau de vie local (Santini, 2006). Ainsi, le même livre présente un coût distinct au Liban, en Égypte, en Syrie, au Maroc et dans les différentes librairies du quartier des Habous. Dans ce domaine, aucune loi spécifique ne contraint les éditeur-distributeurs, libres de fixer leur prix en vue d'être plus compétitifs que leurs concurrents ${ }^{20}$. $\mathrm{Au}$ Maroc, leurs efforts se concentrent surtout sur le Coran dont la vente représente, on l'a dit, une part importante de leur chiffre d'affaires. Pour mieux contrôler les coûts de production du Coran et baisser son prix au maximum, les éditeurs du quartier des Habous sont de plus en plus nombreux à fabriquer leur propre modèle de Livre saint.

Le récent «boom» du Coran a même entraîné la création de nouvelles maisons d'édition dans le quartier des Habous, facilitée par l'absence de cadre juridique et de taxation sur le livre culturel au Maroc ${ }^{21}$. Les éditeurs à la tête de ces structures sont généralement d'anciens employés des librairies du quartier désireux d'établir leur propre commerce aux côtés de leurs anciens patrons et de leurs clientèles. Ces jeunes professionnels - âgés d'une trentaine d'années en moyenne - n'ont le plus souvent pas une bonne connaissance du Coran. Leur choix d'éditer le Livre saint est avant tout «commercial », comme ils disent. Ils sont des «bisnessmen », ajoutent-ils, à la recherche d'un bénéfice économique plus que symbolique; même si certains se targuent de véhiculer la parole divine et d'être de bons musulmans.

Pour fabriquer le Livre saint, les éditeurs ont recours à différentes options, plus ou moins coûteuses. La plus économique consiste à scanner les pages d'un Coran généralement conçu en Égypte ou au Liban de manière à élaborer un nouvel encadrement (ornementations, format, mise en pages, voir fig. 1 et 2) et créer un mushaf différent de celui copié. Tandis que les moyennes et grandes structures sous-traitent ce travail de recomposition typographique à leurs collègues égyptiens et libanais spécialisés dans ce domaine, les petits éditeurs réalisent eux-mêmes ces modifications par souci

20 Alors qu'en France, par exemple, la loi sur le prix unique du livre (1982) oblige toute personne qui publie ou importe un livre à fixer un prix de vente public que doivent respecter les détaillants de l'ensemble du territoire national.

21 Seul l'article 1 du Code de la presse, figé depuis 1973, régit l'édition et la distribution. L'éditeur-distributeur doit simplement être inscrit au registre du commerce et avoir un numéro de patente. Le Code de la propriété culturelle et artistique, élaboré en 1971, concerne seulement les domaines cinématographique et musical (De Blic, 2002). 


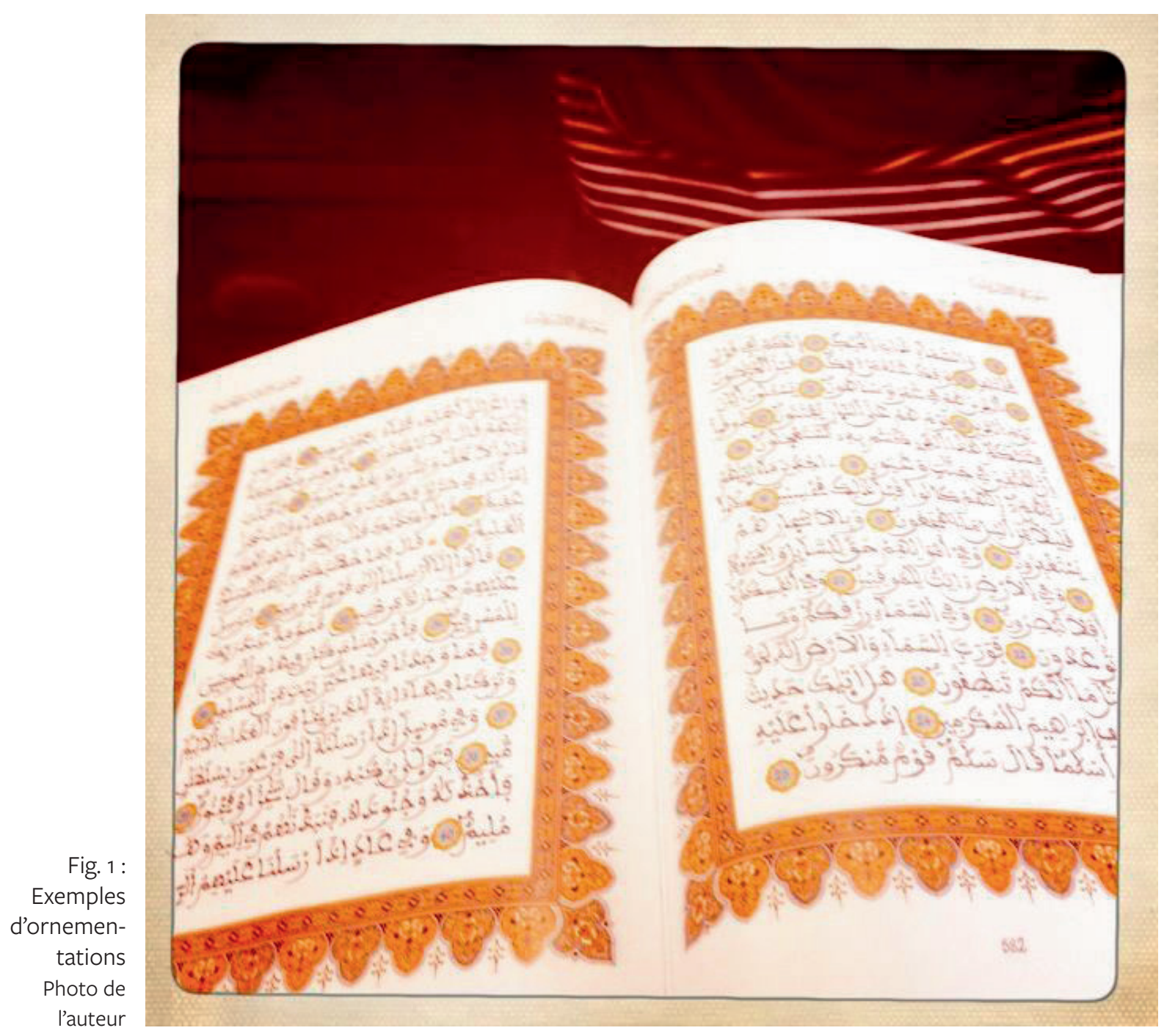

d'économie. L'utilisation de logiciels de PAO (publication assistée par ordinateur) basiques comme InDesign ou Quark XPress leur permet de redéfinir facilement les compositions végétales qui encadrent le texte ou même d'importer, sur chaque page du livre, des modèles d'arabesques prédéfinis.

Les éditeurs qui souhaitent changer le format du livre ou créer de nouveaux modèles tels que les livres de Coran commentés (où apparaissent, en marge, les explications des mots et des passages difficiles) sont contraints de «saisir» le texte sur ordinateur, c'est-à-dire de le réécrire intégralement pour pouvoir retravailler plus aisément la mise en pages. Soucieux de s'adapter aux besoins de leur public cherchant, on l'a dit, à apprendre la parole divine, beaucoup d'éditeurs élaborent des modèles de Coran à caractère didactique, dont la composition typographique vise à faciliter la 
Fig. 2 : Autres exemples d'ornementations Photo de l'auteur

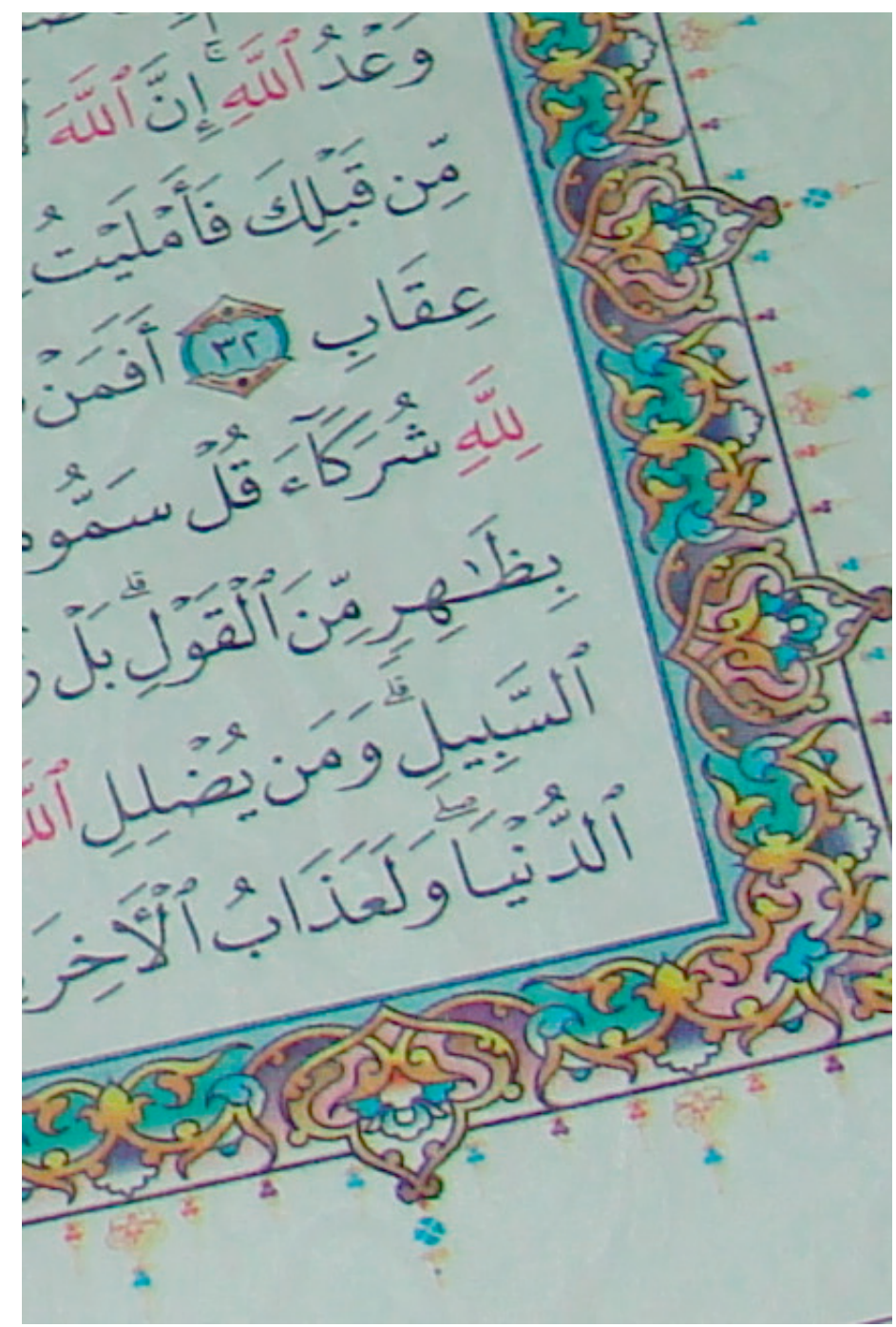

mémorisation visuelle des versets. Dans ce but, les pages de certains Livres saints s'arrêtent à la fin des versets plutôt qu'en leur milieu. Un autre procédé visant le même objectif mnémotechnique fait apparaître un hizb ${ }^{22}$ (c'est-à-dire un soixantième du Coran) sur chaque page. De cette façon, le fidèle, tenu de lire intégralement le Coran (composé de 6o hizb) chaque mois, sait plus facilement où il en est dans sa lecture.

22 Hizb : «Signifie en premier lieu "groupe, faction, ensemble des partisans d’un homme qui partagent ses idées et sont prêts à le défendre”. II signifie également "part, portion” et c'est en partant de cette acception qu’il en est venu à désigner une division du Coran ainsi qu’un ensemble de formules liturgiques» (Macdonald, 1971). Certaines divisions du Coran sont devenues plus populaires que d'autres, notamment les trentièmes (juz') et les soixantièmes (hizb). 
Il existe même des modèles de Coran divisés en plusieurs livres - généralement six composés chacun de cinq hizb - rangés dans une valise. En plus d'aider à la mémorisation du texte, ce découpage permet aux personnes souhaitant le lire durant la journée, aux moments de pause, de transporter un seul petit exemplaire plutôt que l'ouvrage tout entier.

Ces différentes stratégies éditoriales visent deux objectifs : d'une part, concevoir le prochain best-seller du quartier; d'autre part, contourner les accusations de «piratage », comme disent les éditeurs ${ }^{23}$.

\section{Des corans «frauduleux»?}

Mais que signifie «pirater» dans le cas du Coran, qui représente un texte unique : la Parole de Dieu. «Pirater» par rapport à quel «original»?

Alors que la Bible est soumise aux droits d'auteurs (plus précisément de traducteurs) classiques, le texte coranique ne répond à aucune réglementation officielle dans ce domaine. Selon un éditeur du quartier :

Les droits d'auteur, ça n'existe pas dans le Coran. Tu vas pas payer au bon Dieu des droits d'auteur, l'auteur c'est qui? C'est le bon Dieu, donc tu ne paies pas de droits d'auteur ${ }^{24}$.

Il existe cependant des «droits de production et d'écriture » qui protègent les caractéristiques physiques spécifiques de l'objet conçues par l'éditeur telles que les enluminures (tadhîb) ou encore la calligraphie (khâtt) dans le cas où il aurait fait appel au service d'un calligraphe. Cette notion de «droits de production et d'écriture» indique que l'originalité d'un mushaf réside avant tout dans ses propriétés matérielles et esthétiques, voire aspectuelles. Au Maroc, suivant le type de livre de Coran produit, conforme à la lecture de Warsh ou à la lecture de Hafs - dont les variantes concernent «des points de vocalisation, des emplacements de pauses dans la récitation ou de fins de versets ainsi que des détails de prononciation » (Moezzi, 2007)25 - cette singularité

23 Notons ici que, jusqu'à une période récente, la traduction du Coran suscite peu l'intérêt des éditeurs marocains dont la clientèle cherche avant tout à l'apprendre en arabe, langue de la Révélation. Ces dernières années, néanmoins, de plus en plus de traductions de Coran sont en vente dans les librairies des Habous : en français, anglais, espagnol, allemand, hollandais et même en berbère. Sur ce dernier point, voir Pouessel (2008).

24 Entretien du 8 octobre 2012 à Casablanca.

25 Hafs et Warsh sont l'appellation donnée à deux des sept lectures coraniques (qirấa). «Le mot lecture rend de manière imparfaite le terme technique arabe de qirâa. Il s'agit ici de façons divergentes de rendre (réciter) le texte coranique initialement par oral, par la suite aussi par écrit, sans que les divergences entre les différentes "lectures" affectent en rien la conviction des musulmans que toutes celles qui sont canoniques ont la même valeur. À partir du iv/ $x^{e}$ siècle, ces dernières sont au nombre de sept, auxquelles s'en ajouteront sept autres. [...]. Trois variantes s'imposèrent au fil des temps, celle de Nâfi' transmise par Warsh, celle de 'Âsim trans- 
repose davantage sur les ornementations ou sur le style calligraphique. Alors que les modèles de Coran Hafs sont le plus souvent reproduits en caractères mobiles suivant la calligraphie naskhi, les modèles de Coran Warsh présentent majoritairement une écriture manuscrite ${ }^{26}$ en calligraphie maghribî. Pour cette raison, les exemplaires de Coran Hafs ne sont généralement pas concernés par les «droits d'écriture (huqûq al-kitâba). La singularité de ce type de mushaf ne réside pas tant dans sa calligraphie (informatique et standardisée) que dans les propriétés esthétiques du livre comme les enluminures ou les matériaux - notamment les pierres précieuses - enrichissant les couvertures et les coffrets, etc. Pour ce qui est des livres de Coran Warsh, en revanche, «c'est l'écriture qui change», déclare un éditeur, poursuivant «elle est plus ou moins jolie, bien faite ${ }^{27} »$.

C'est ce modèle que les libraires des Habous déclarent vendre le plus, surtout aux personnes âgées d'une quarantaine d'années (et plus) ayant appris à lire le Coran dans le kuttâb au moyen de la planchette en bois. En outre, c'est le Coran Warsh que les donateurs cherchent à acquérir en vue de l'offrir aux mosquées qui respectent généralement le rite malékite souvent associé à ce type de lecture. Les exemplaires de Coran Hafs sont quant à eux achetés par quelques clients cherchant à « décorer » leurs intérieurs avec de beaux modèles de Coran, déclare un éditeur. "Comme ils ne les achètent pas pour lire, ils s'en fichent», poursuit-il. À l'exception des jeunes générations qui utilisent davantage le Coran Hafs, plus lisible à cause des caractères d'imprimerie auxquels elles se sont familiarisées à travers les journaux, les manuels scolaires et les livres de littérature générale. Toutefois, cette dernière catégorie de lecteurs ne représente pas encore une clientèle importante des librairies des Habous.

La préférence d'une majorité de fidèles pour le Coran de lecture Warsh motive les éditeurs à fabriquer prioritairement cette catégorie d'ouvrages. Un processus confronté à des contraintes spécifiques : alors que le livre du Coran Hafs est, on l'a dit, libre de droits d'écriture (et non de production), le livre du Coran Warsh est protégé par la double réglementation.

Jusqu’à une période récente, les droits d'écriture du Coran Warsh n'appartenaient qu'à une seule maison d'édition, Dâr al- mushaf al-sharîf, installée au Caire. Dans les faits, les éditeurs des Habous ont le choix entre deux options, bien résumées par l'un d'entre eux : «Soit tu achètes les droits de celui qui a écrit le Coran, à lui (le

mise par Hafs et celle de Abû 'Amr transmise par al-Durri» (Moezzi, 2007, p. 814). Aujourd'hui, à l'exception de larges territoires d’Afrique occidentale et centrale où la lecture de Warsh, généralement associée au rite malékite est répandue, c'est la lecture de Hafs qui, dans le reste du monde musulman, est la plus connue. Sur les qirâat voir Larcher (2013), Blachère (2001) et Brockett (1984).

26 L'usage de ce pléonasme sert à souligner la différence entre caractères d'imprimerie et caractères manuscrits.

27 Entretien du 8 octobre 2012 à Casablanca. 

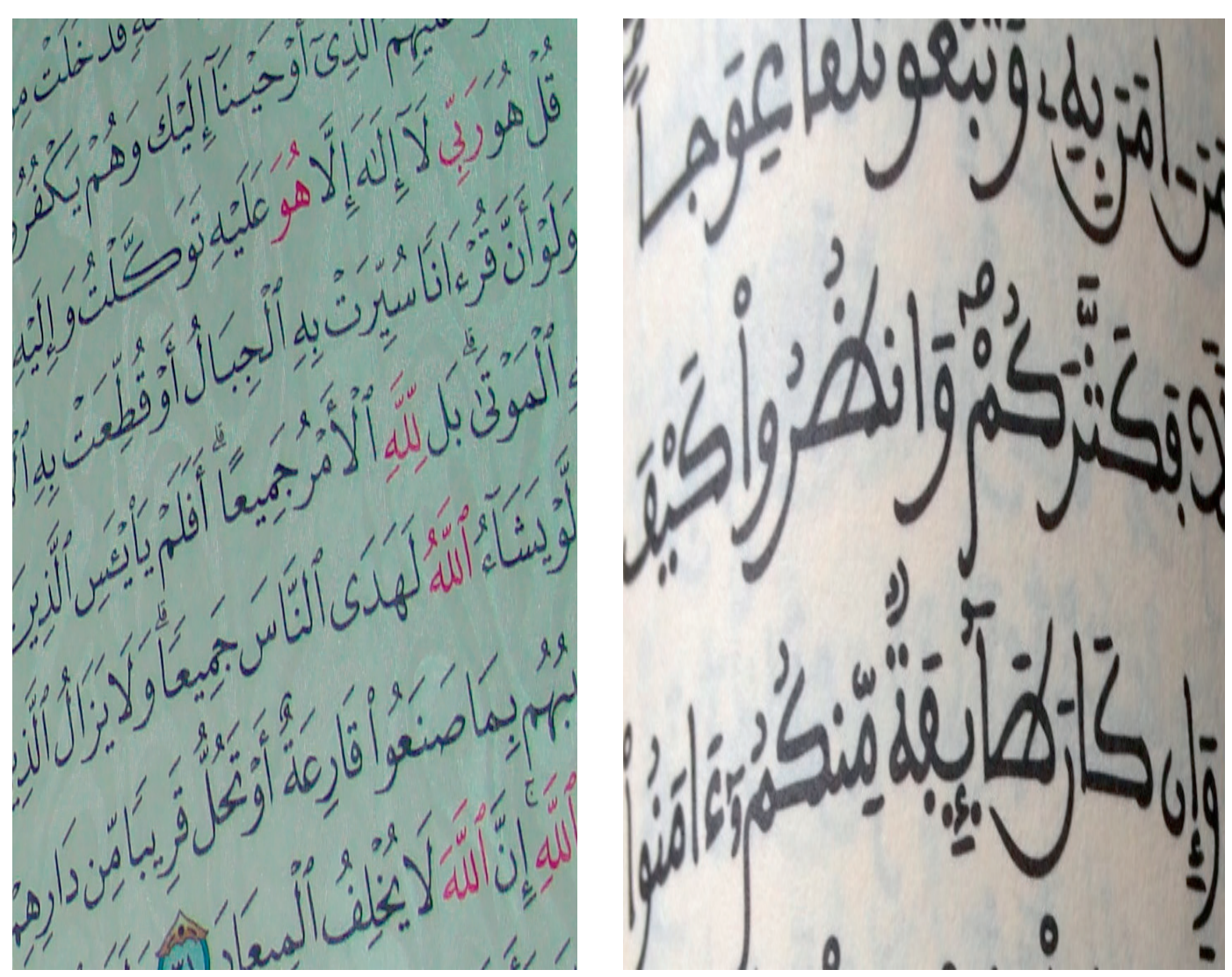

Fig. 3 : Écriture typographique et écriture manuscrite

calligraphe) ou à l'éditeur qui les lui a achetés, soit tu fais ton propre Coran en ramenant un calligraphe pour qu'il te fasse ton écriture, à toi ${ }^{28}$."

La dernière option consiste en un processus long et coûteux que seules les grosses structures éditrices peuvent se permettre de réaliser. Les petits éditeurs, au faible capital, «piratent » quant à eux l'écriture du Coran conçu par Dâr al-mushaf al-sharîf ${ }^{29}$. Pour ce faire, ils utilisent les mêmes procédés que ceux décrits précédemment : ils scannent les pages du livre, en modifient parfois les décorations à partir de logiciels PAO, puis envoient le fichier informatique terminé à l'étranger pour impression.

28 Entretien du 6 octobre 2012 à Casablanca.

29 Notons que des années auparavant, Dâr al-mushaf al-sharîf avait procédé aux mêmes manipulations que ces éditeurs puisque «l'écriture» dont elle est la propriétaire est celle d'un calligraphe et faqîh (juriste) marocain. Selon le professeur Maghouazi, spécialiste des arts traditionnels au Maroc, un éditeur qui avait racheté la copie lithographiée directement au calligraphe l’a fait imprimer en Égypte mieux équipée dans ce domaine. C'était une pratique fréquente entre les années 1955 et 1985 : à cause du développement tardif de l'imprimerie marocaine, les éditeurs n'avaient généralement d’autres choix que d’externaliser la production à l'étranger, en particulier au Caire. Ces observations contribuent à expliquer pourquoi les droits d'écriture du Coran Warsh appartiennent à une maison d'édition égyptienne (entretien du 16 avril 2013 à Rabat). 
Suivant les cas, les éditeurs prennent plus ou moins de précautions, sachant que moins ils en prennent, plus ils augmentent leur productivité et réduisent le coût du livre à l'unité. Pourtant, le processus d'estampille du Livre saint est en principe très strict : des tirages entiers ont déjà été détruits parce qu'on y avait décelé quelques erreurs ou omissions. Ce souci d'exactitude est lié et s'étend à la récitation du Coran. Il implique un contrôle à chaque phase de production. À la fin du processus, un «certificat» (shahâda) doit normalement être délivré par des spécialistes en sciences coraniques : des oulémas indépendants ou membres d'une institution islamique, rémunérés pour leur travail ${ }^{30}$. Néanmoins, par souci d'économies, beaucoup d'éditeurs n'effectuent pas ces contrôles dans leur intégralité. Si bien que le Coran est de plus en plus reproduit avec des erreurs. Celles-ci sont de différents ordres, expliquent les éditeurs : d'une part, il y a les erreurs liées aux conditions techniques d'impression - pages manquantes ou désordonnées -, d'autre part, celles en relation avec les méthodes de lecture coranique Hafs et Warsh mélangées dans certaines éditions, en particulier celles «saisies » à l'ordinateur. On y trouve certaines sourates qui respectent les règles de récitation de Hafs et d'autres les règles de récitation de Warsh.

\section{Le mécontentement des fidèles}

C'est actuellement une polémique vive au Maroc : les journaux locaux ne cessent d'alerter la population sur la circulation croissante de livres de Coran harâm (illicite). J'ai moi-même, en tant que libraire, entendu un nombre important de clients dénoncer ces pratiques. Pour certains, elles sont le fait de «groupes musulmans fondamentalistes » préoccupés de produire au plus vite un très grand nombre d'exemplaires à un prix compétitif; pour d'autres, ce sont «les Américains » qui sont à l'origine du blasphème : «Ils le font exprès », déclare une cliente, poursuivant : «Ils changent le message de Dieu pour nous induire en erreur.» Cet éditeur, quant à lui, accuse «les imprimeries catholiques du Liban : ce qui les intéresse, déclare-t-il, c'est d'avoir l'argent. Le côté spirituel, ils s'en fichent».

Des clients mécontents n'hésitent pas à se rendre dans le quartier des Habous pour désigner aux commerçants les corans frauduleux acquis dans leurs boutiques. Certains d'entre eux profitent de l'occasion pour indiquer les erreurs commises sur le texte, une manière pour ces fidèle de montrer leur connaissance du Coran. Ces clients sont imams, muezzins (personnel de la mosquée chargé de lancer l'appel à la prière), professeurs et étudiants en droit islamique à l'université ou simples fidèles souhaitant montrer leur piété. Les plus rigoristes d'entre eux vont parfois directement au ministère des Habous et des Affaires islamiques ou dans ses délégations régionales

30 Pour une étude plus approfondie de l'estampille du Livre saint au Maroc et du rôle des oulémas dans ce processus, se reporter à Cohen (2015, à paraître). 
pour dénoncer les erreurs et attirer l'attention des services publics. Quelques-uns exigent même la condamnation des éditeurs ayant manqué de rigueur sans jamais toutefois entreprendre de procédures judiciaires. À ces dénonciations, s'ajoute la critique souvent sévère de mouvements religieux qui ne manquent pas de reprocher au roi son laxisme. Pour de nombreux animateurs de blogs et forums de discussion décrits comme "islamistes », la circulation d'exemplaires de Coran harâm est la preuve que Mohammed VI manque à son rôle de garant du respect de l'islam. Un argument au cœur des campagnes et/ou actions menées par les deux organisations qui dominent la scène politique islamiste marocaine (Tozy, 1999) : Islah wa al-Tawhid (Réforme et unicité), devenu en 1997 le Parti de la Justice et du développement (PJD, aujourd'hui principale force d'opposition au Parlement), et Al-'Adl wa al-Ihsân (Équité et don de soi) ${ }^{31}$.

Devant l'influence grandissante de ces groupes, le royaume a dû prendre ses dispositions.

\section{Pour un seul Livre : le «Coran marocain}

\section{» !La construction d'un islam national par le Livre}

D'une part, les contrôles exercés dans le quartier des Habous et aux ports où arrivent les ouvrages en provenance du Moyen-Orient sont plus stricts et plus fréquents. Depuis 2010, le mushaf répond à une réglementation spécifique. Les copies importées ne sont plus vérifiées par le «service de lecture des ouvrages » du ministère de la Communication et l'Information mais par celui des Habous et des Affaires islamiques ${ }^{32}$. Plus que de limiter le nombre de «corans falsifiés», suivant l'expression employée dans le dahîr (décret royal) sur la création de la Fondation Mohammed-VI pour l'édition du saint Coran, la mise en place d'une procédure de vérification spécifique au Coran vise à souligner la spécificité de ce livre et à mieux connaître le marché local à travers la production systématique de statistiques. L'État peut désormais mesurer le nombre exact d'exemplaires de Coran importés dans le pays, en connaître la provenance et la lecture (Warsh ou Hafs) à laquelle ils se conforment. Alors que sous le règne d'Hassan II (1963-1999), les ouvrages visés par la censure portent principalement sur le roi, le Sahara occidental et les droits de l'homme, aujourd'hui, elle concerne surtout

31 Al-'Adl wa al-Ihsân, dirigé par Abdeslam Yassine, représente un courant important de l'islam marocain dont l'action politique cible des couches populaires les plus défavorisées. Ses actions privilégient la dimension caritative et l'éducation religieuse et scolaire avec une forte moralisation de la société. Organisé sous forme d’association, il ne tient pas encore, à l'instar du PJD, à se transformer en parti politique.

32 Seuls les livres de Coran rapportés par les pèlerins échappent à ce contrôle. Ils sont pourtant en nombre grandissant. Grâce à l'élévation du niveau de vie, les fidèles sont de plus en plus nombreux à se rendre à La Mecque pour réaliser le pèlerinage (hâjj), d’où ils ne manquent pas de ramener un ou plusieurs exemplaire(s) de Coran pour leur propre usage ou pour les offrir. À ce sujet, voir Hammoudi (2005). 
les livres islamiques. Cette observation est corroborée par l'interdiction d'un nombre croissant d'éditeurs orientaux aux dernières éditions du Salon international de l'édition et du livre de Casablanca (SIEL).

D’autre part, le ministère des Habous et des Affaires islamiques a annoncé en 2005 la création de la Fondation Mohammed-VI pour l'édition du saint Coran :

[Elle] sera compétente à titre exclusif pour procéder aux opérations d'enregistrement, d'impression, de publication et de distribution du Livre saint avec tout ce qu'elles nécessitent en termes de vérification et de précision dans sa transcription et sa lecture. Cette fondation veillera à garantir l'exactitude des copies du Livre saint en circulation dans [le] Royaume et ce, en autorisant leur impression ou leur distribution pour s'assurer qu'elles sont exemptes de toute erreur et de toute falsification (Dahîr sur la création de la Fondation Mohammed-VI pour l'édition du saint Coran).

Composée d'un directeur (ancien professeur de droit islamique à l'université), d'un «conseil d'administration » et d'un « organe scientifique » regroupant des «personnalités spécialisées dans les sciences du Coran et des experts spécialisés dans l'informatique et les arts de la calligraphie et de l'impression » (op. cit.), la Fondation Mohammed-VI a non seulement pour mission d'encadrer l'édition et la distribution du Livre saint dans le royaume mais aussi de «reproduire sur ordre de [Sa] Majesté le saint Coran sur la base de la narration de Warsh tenue de Nafiâ selon les règles adoptées dans les sciences des transcriptions et des lectures» (op. cit.). Un «Coran uniformisé », «marocain », «national », autant de qualificatifs employés dans les journaux étroitement liés au Palais pour saluer l'initiative du roi vue comme «une nouvelle illustration de son intérêt constant pour le livre sacré33». À l'approche des élections législatives en 2006, on peut déceler dans la réponse du pouvoir le signe d'une inquiétude : la crainte que le Parti islamiste de la justice et du développement (PJD) devienne la première formation politique au Parlement. L'État s'est positionné de façon claire pour signifier qu'il s'efforce de garantir le respect de l'islam. Un prétexte qui aurait pu sinon alimenter la campagne politique du PJD, déjà ciblée sur le recul des valeurs religieuses dans le pays.

33 Voir file:///Fondation\%2OMohammed\%20VI\%20-\%20Coran\%20-\%20Edition\%20-\%20Maroc.webarchive

34 Professeur à l'université de Rabat, il fut directeur de l'Institut d'Études africaines puis directeur de la Bibliothèque nationale du Royaume et auteur de plusieurs romans écrits en arabe. 
prédécesseurs, généralement alim (singulier d'oulémas). À la suite des attentats du 11 septembre 2001 et surtout de ceux de Casablanca en 200335, Ahmed Tawfiq met en place une nouvelle politique publique religieuse visant à lutter contre la montée des extrémismes. Il défend la promotion d'un islam national en ancrant mieux ses spécificités dans la vie des Marocains. Ces spécificités sont au nombre de trois : le rite malékite, le soufisme et l'ouverture. Construites à travers l'histoire du pays, ces caractéristiques ont contribué à définir les contours d'un islam national qu'il s'agit aujourd'hui de (re)valoriser dans le but de contrer l'influence grandissante d'autres rites musulmans, en particulier hanbalite plus rigoriste et proche du wahhabisme promu par certains mouvements islamistes ${ }^{36}$. Le roi s'est exprimé clairement sur ce point :

Depuis quatorze siècles, les Marocains ont choisi d'adopter l'islam parce que religion du juste milieu, il repose sur la tolérance, honore la dignité de l'homme, prône la coexistence et récuse l'agression, l'extrémisme et la quête du pouvoir par le biais de la religion. C'est à la lumière de ces enseignements que nos ancêtres ont édifié une civilisation islamique et un État indépendant du Califat du Machrek [Orient arabe], se distinguant par son attachement à la commanderie unique des croyants, par son ouverture en matière de culte et par l'exclusivité du rite malékite. [...] Est-il donc besoin pour le peuple marocain, fort de l'unicité de son rite religieux et de l'authenticité de sa civilisation d'importer des rites cultuels étrangers à ses traditions? Nous ne le tolérerons pas d'autant plus que ces doctrines sont incompatibles avec l'identité marocaine spécifique. À ceux qui s'aviseraient de se faire les promoteurs d'un rite étranger à Notre peuple (Discours du trône, 30 juillet 2003) (Tozy, 1999).

La lutte du royaume contre les mouvements décrits comme «salafistes » et «intégristes» doit donc passer pour le roi et son ministre des Affaires islamiques par l'affirmation d'une identité religieuse propre fondée sur la fabrication d'un islam «marocain» «authentique». Le Livre est un instrument central de cette politique.

\section{Le livre du Coran au cœur d'une politique d'«authenticité»?}

Inaugurée en 2010, la Fondation Mohammed-VI pour l'édition du saint Coran édite un mushaf caractérisé par la lecture de Warsh et la calligraphie maroco-andalouse, encore appelée maghribî. Développée au $\mathrm{X}^{\mathrm{e}}$ siècle au Maghreb et en Espagne dans le but de remplacer les styles «classiques » issus des territoires centraux du califat (en particulier

Série d'attentats suicides qui ont provoqué la mort de 45 personnes et une centaine de blessés.

36 Dans son ouvrage Les islamistes marocains. Le défi de la monarchie, Malika Zeghal distingue trois types d'islamisme : l'un «légalisé», représenté par le Parti de la Justice et du développement; l'autre «mystique et rebelle», représenté par l'association Justice et Bienfaisance; le dernier «salafiste/jihâdiste», lié aux réseaux internationaux d’un islam mondialisé (Zeghal, 2005). 
le naskhi) et élaborer une «identité graphique» propre à la région (Moezzi, 2007), le maghribî est une fois de plus utilisé par la monarchie (ancien sultanat) comme une marque de distinction. Elle est l'expression d'une marocanité archaïque à sauvegarder par la mise en valeur d'anciens manuscrits coraniques considérés comme authentiques et à ce titre exposés à la Bibliothèque nationale du royaume du Maroc et dans les vitrines du hall de la fondation.

Outre la calligraphie maroco-andalouse, le Coran national - nommé mushafmuhammadî en référence au roi - se distingue par une tradition d'enluminures définie par la fondation comme proprement marocaine. Une «marocanité» visible, explique le directeur, dans les formes géométriques et les couleurs des compositions végétales encadrant le texte coranique (fig. 4 et 5). Autant de particularités qu'il est aussi possible de repérer dans les décorations qui enrichissent les mosquées du royaume ${ }^{37}$.

Une employée de la Fondation Mohammed-VI chargée de la mise en page du mushaf muhammadî précise : «On veut que ça rappelle le goût des Marocains.» «On veut que ça reflète la culture marocaine ${ }^{38} »$, ajoute le directeur. Tant la calligraphie que les enluminures du mushaf muhammadî visent à évoquer et ranimer le patrimoine religieux et artistique local.

D’ailleurs le calligraphe et l'enlumineur nommés par Ahmed Tawfiq pour réaliser le mushaf muhammadîne sont autres que Mohamed Maalmine et Abdellah El Ouazzan. Célèbres au Maroc pour la maîtrise de leur art, ils ont tous deux travaillé aux services du roi Hassan II et Mohammed VI pour la publication des discours royaux et dahîr ainsi que pour la décoration de certains monuments publics. Ils sont des figures centrales du patrimoine artistique marocain, reconnus pour être les meilleurs spécialistes des anciens manuscrits coraniques du pays ayant servi de modèle à la réalisation du mushaf muhammadî. Leur connaissance de la calligraphie a été complétée de celles d'oulémas spécialistes des sciences du Coran et des différentes lectures. Au cours de la préparation du manuscrit, ces «experts » d'un autre type ont eu pour charge de vérifier le respect des règles calligraphiques maghribî et de la narration de Warsh. Après de nombreux allers-retours avec le calligraphe, une fois que les oulémas ont été assurés de l'exactitude du manuscrit, l'enlumineur a pu réaliser les ornementations qui encadrent le texte sur chaque page du livre. Le texte, composé de plusieurs feuilles blanches de grand format (A3), a par la suite été scanné et retravaillé sur ordinateur par les graphistes de la fondation, de manière à redéfinir le format, souligner les contours des lettres et raviver les couleurs des ornementations. Ce n'est qu'après ces différentes

37 Les mosquées du royaume ont été récemment placées au cœur d’une exposition organisée par le ministère des Habous et des Affaires islamiques. Voir «Inauguration de l'exposition "Mosquées marocaines à travers l'histoire" », Fondation de la Mosquée Hassan II, http://fmh2.ma/fr/activites-damir-al-mouminine/51inauguration-de-lexposition-Imosquees-marocaines-a-travers-Ihistoirer-.html

38 Entretien du 12 avril 2013 à Mohammedia. 


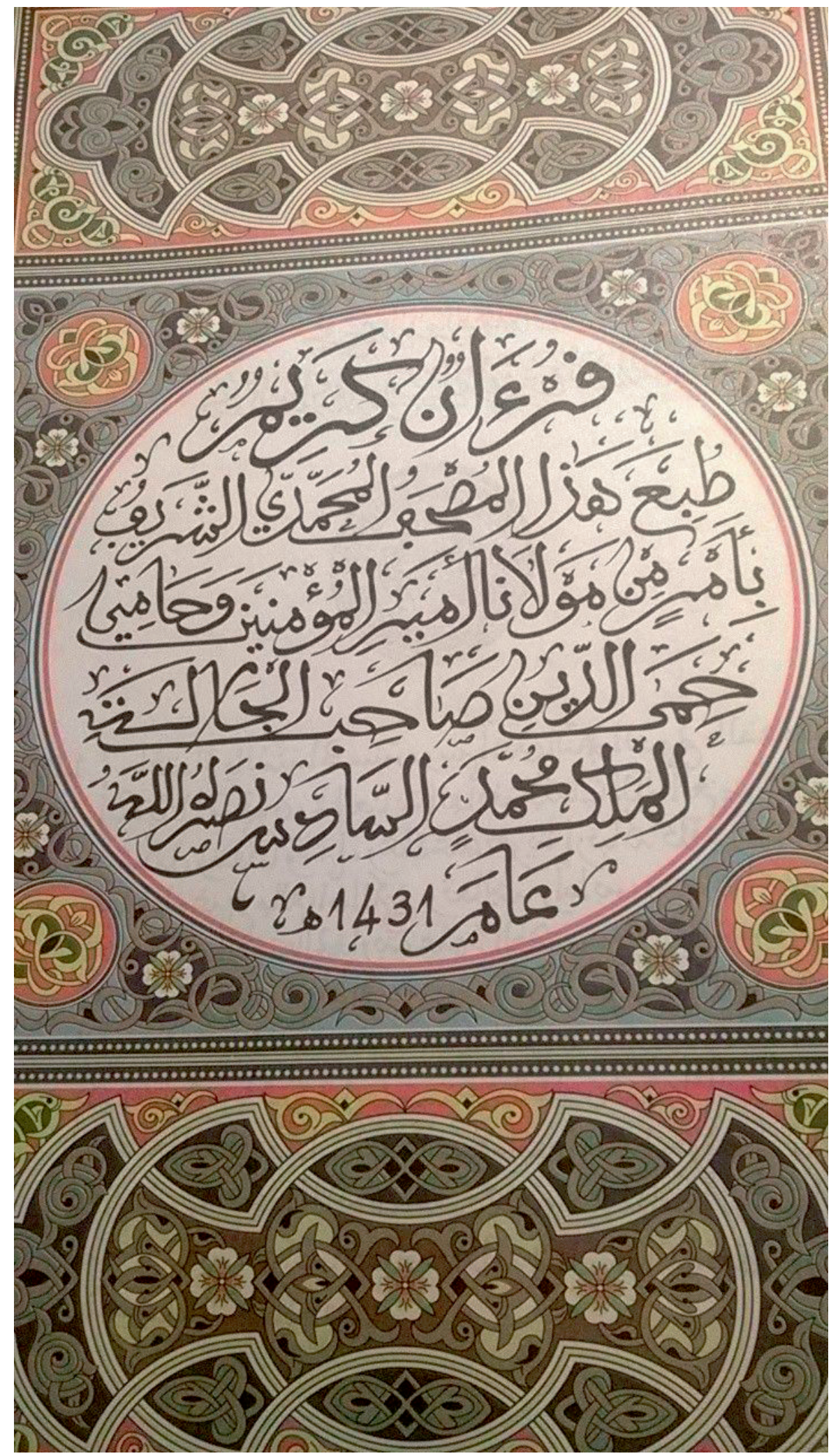

Fig. 4 : Enluminures figurant sur la troisième page du mushaf muhammadî 


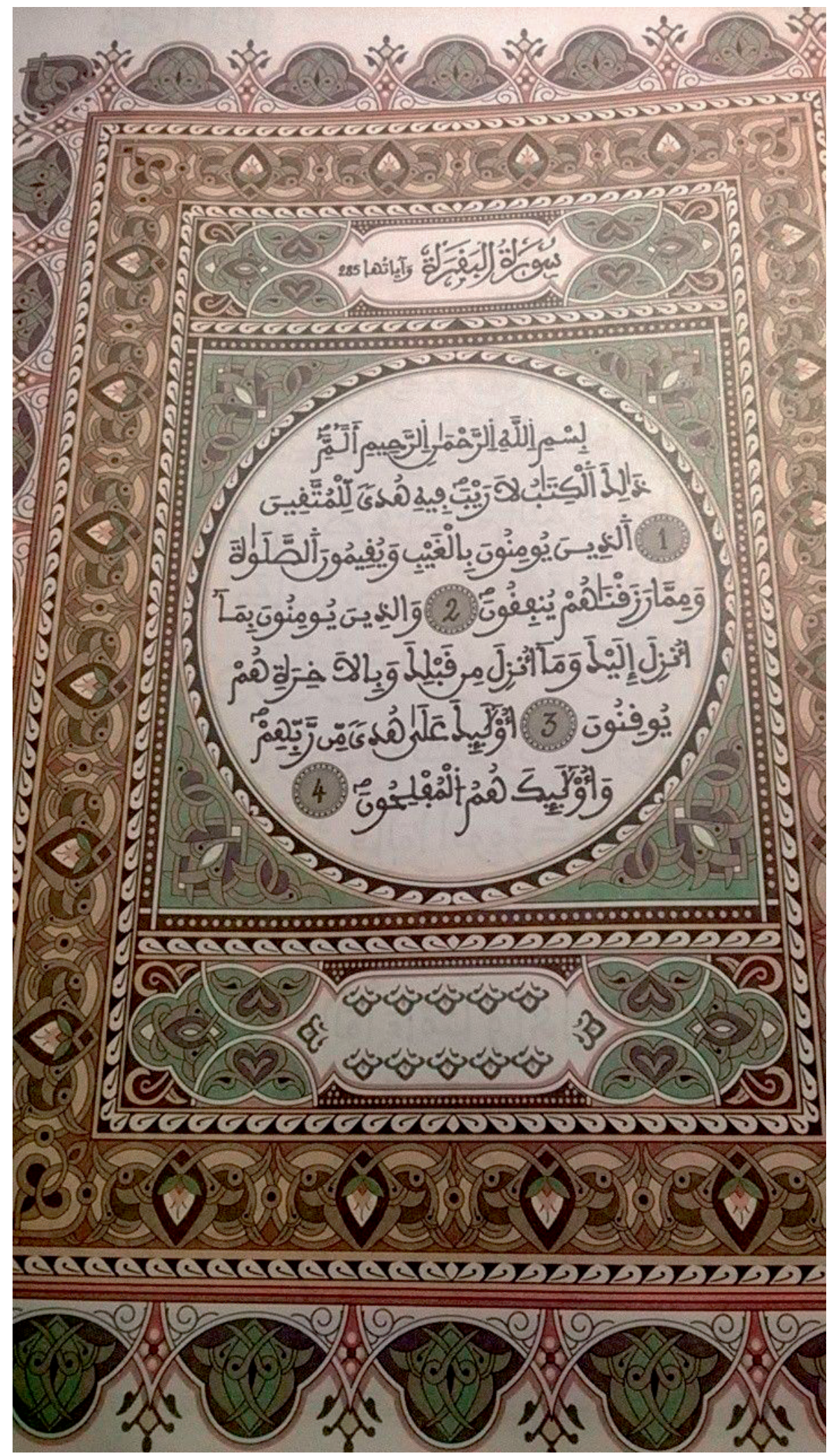

Fig. 5 : Autre exemple d'enluminures apparaissant en page 8 du mushaf muhammadî 

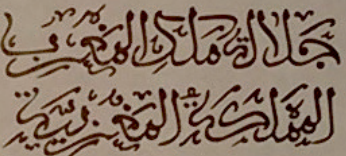

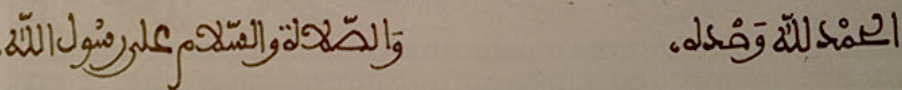

Fig. 6 : Le cachet du roi sur la quatrième page du mushaf muhammadî

Page suivante:

Fig. 7 : Certificat (shahâda) à la fin du mushaf muhammadî
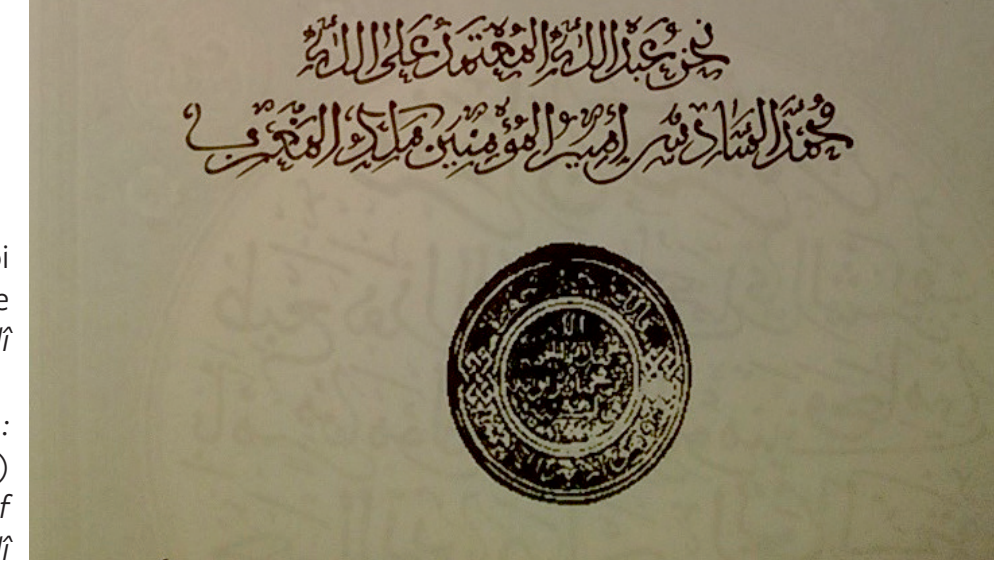

étapes et une ultime vérification par les oulémas que le mushaf muhammadî a été imprimé à l'imprimerie royale de Mohammedia, ré-ouverte pour l'occasion et équipée de nouvelles machines achetées en Allemagne de manière à assurer une reproduction infaillible et de qualité.

On peut donc parler d'une «nationalisation » et d'une «patrimonialisation », voire d'une «autochtonisation » des chaînes de production du Coran ${ }^{39}$. L'objectif du ministre des Habous et des Affaires islamiques est de produire un Coran spécifiquement marocain dont la matérialité est construite en référence à une tradition ancienne de calligraphie et d'enluminures qui est à ce titre, on l'a dit, patrimonialisée. Dans ce double processus, le manuscrit est promu comme gage d'authenticité et d'originalité en vue de garantir un mushaf muhammadî «halal» (licite) produit dans l'imitation des manuscrits marocains des origines. Cette forme de «labellisation» du Coran national est soulignée et renforcée par le cachet du roi et par la signature des oulémas membres de l'organe scientifique de la fondation.

39 Un article en cours de préparation sur la fabrication du mushaf muhammadî étudie en détail ces procédures de «nationalisation» et de «patrimonialisation» en portant l'attention à la manière dont elles sont utilisées par le royaume pour tenter de mieux contrôler la gestion du culte et la transmission du Coran aux fidèles (Cohen, 2015, à paraître). 


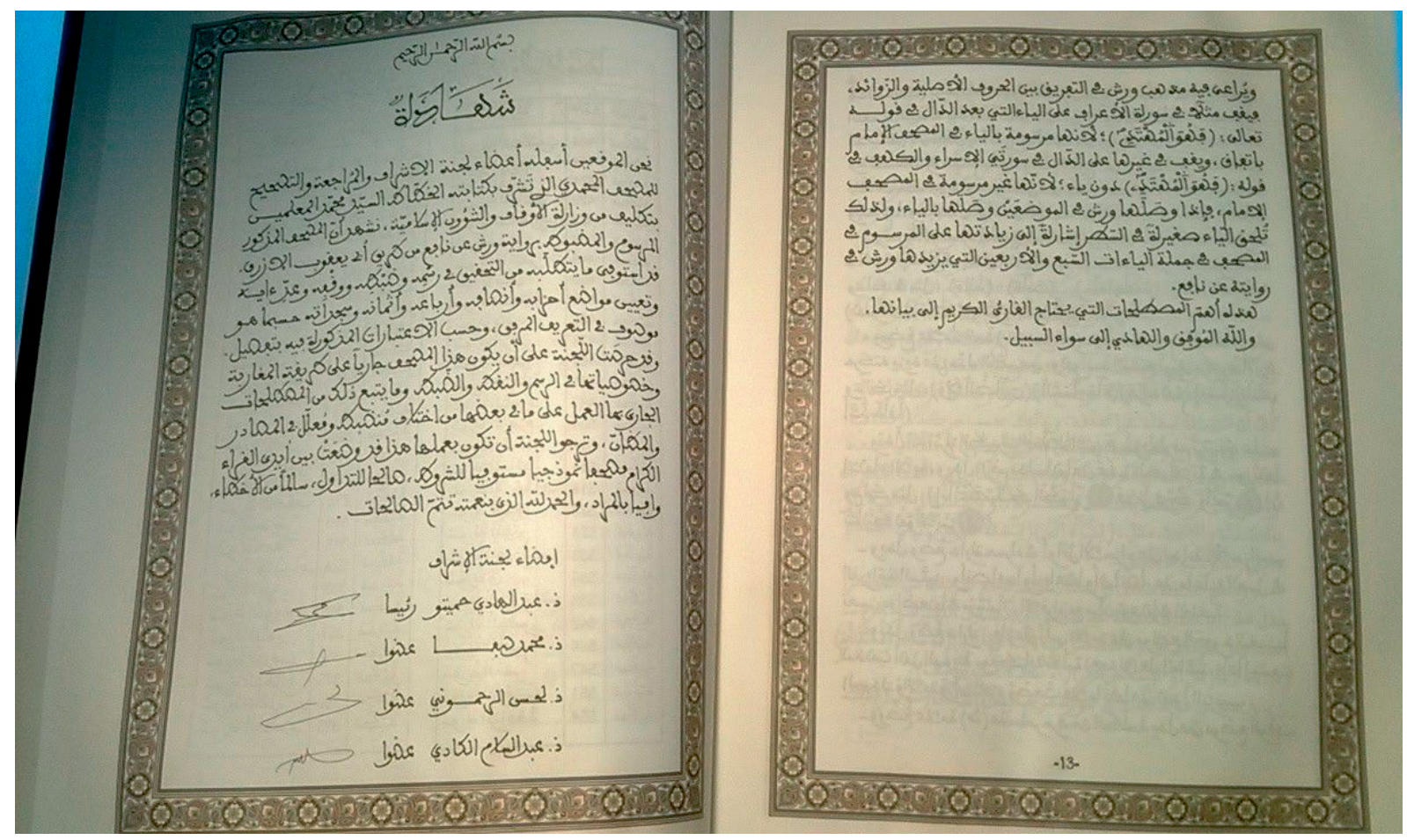

\section{L'imposition du Livre unique et la fin d'un marché}

Cette tentative d'authentification du «Coran marocain » conduit à une confusion entre l'imprimé et l'écrit. Le statut du mushaf muhammadî se situe à la frontière entre le livre et le manuscrit ${ }^{40}$. Statut auquel les éditeurs sollicités par la fondation doivent se tenir en respectant à la lettre les caractéristiques de l'édition nationale décrites plus haut. C'est en janvier 2013 que la fondation, pour répondre à l'objectif $2013 \mathrm{du}$ «million d'exemplaires », a lancé son premier appel d'offres destiné aux professionnels du secteur. Le processus à suivre par les éditeurs intéressés est simple : ils doivent acheter «les droits d'écriture et de production» du mushaf muhammadî en versant la somme de 15000 dh à la fondation pour chaque année commerciale. À cette somme s'ajoutent 3 dh par exemplaire pour vérification par «l'organe scientifique». En outre, les éditeurs sont tenus de respecter le prix unique de 6 o dh fixé par le ministère des Habous et des Affaires islamiques, de manière à «éviter au maximum la concurrence et la spéculation sur le Coran ", explique le directeur de la fondation. Enfin, les éditeurs s'engagent à respecter rigoureusement l'écriture sur le plan morphologique et esthétique

40 Certes, c'est là une caractéristique commune à toutes les éditions modernes de Coran dont l'esthétique reprend globalement celle des anciens manuscrits. Néanmoins, dans le cas du mushaf muhammadî, la ressemblance est poussée à son paroxysme puisque y est reproduite l'écriture manuelle plutôt que les caractères mobiles. 
(formes, couleurs, etc.), la mise en pages et les enluminures du mushaf muhammadî. En outre, ils ne sont autorisés à utiliser qu'un seul type de papier : le papier chamois. Le papier jaune, bon marché et populaire, largement répandu en Afrique, est interdit par la fondation. En revanche, les éditeurs ont la possibilité de produire le mushaf muhammadî en trois différents formats (grand, moyen, petit) et sont libres de concevoir les couvertures et les coffrets de leur choix à condition de ne jamais modifier le prix. Quant aux couleurs ou encore au parfum que certains éditeurs aimeraient ajouter aux pages du mushaf, ils n'y sont pas autorisés. En d'autres termes, si les éditeurs peuvent changer le contenant, c'est-à-dire l'enveloppe matérielle du texte, ils sont dans l'impossibilité de modifier l'écriture et les enluminures, principaux marqueurs d'authenticité du mushaf muhammadî.

C'est le concept même d'éditeur, contraint de renoncer à sa créativité, qui est ici remis en cause par cette entreprise de «nationalisation» du Coran. Cette disposition est en train de reconfigurer le champ éditorial marocain au sens où Bourdieu entend cette notion, comme « un espace relativement autonome - c'est-à-dire capable de retraduire selon sa logique propre toutes les forces externes, économiques et politiques notamment - dans lequel les stratégies éditoriales trouvent leur principe » (1999). Le roi est désormais l'éditeur ( ou le «créateur», pour reprendre l'expression de Bourdieu [ibid.]) exclusif du texte de la Révélation au Maroc. C'est la fin d'un marché florissant pour les commerçants du quartier des Habous. L'un d'entre eux témoigne et se lamente de cette situation, ne manquant pas de grossir le trait:

Le Coran est le livre qui se vend le mieux, c'est clair, mais maintenant c'est terminé tout ça. On ne pourra plus importer le Coran par milliers comme avant, il faudra d'abord le faire contrôler par le ministère des Habous qui verra s'il est correct ou pas, etc. Si on veut importer le Coran maintenant il faudra d'abord déposer sept exemplaires à la commission qui les vérifiera pour voir s'il n'y a pas d'erreurs, etc. Ça [la vérification] coûte 1 dh par page donc si tu ramènes 15000 corans on va dire, tu vas devoir payer $15000 \mathrm{dh}$, qui va payer cette somme? Personne... alors qu'avant il y avait les éditeurs égyptiens et libanais qui venaient nous proposer d'imprimer nos corans et ça faisait tourner le marché, maintenant c'est terminé, il n’y a plus de marché [...]. Maintenant, c'est le royaume qui fait son propre Coran, qui le donne aux mosquées ${ }^{41}$.

Comme cet éditeur, nombreux sont ceux qui, inquiets des risques de la nouvelle réglementation, ont renoncé à concevoir leur propre modèle de Coran. Par conséquent, une seule édition du Coran dominera bientôt les étalages des librairies du quartier et les mosquées : le mushaf muhammadî. 


\section{Pour un seul mushaf : celui du roi}

Pour le ministère des Habous et des Affaires islamiques, l'enjeu principal de cette politique publique religieuse est le lieu de culte. Les milliers d'exemplaires du mushaf muhammadî produits par la fondation et les éditeurs sont destinés à approvisionner les mosquées du royaume qui ne doivent désormais utiliser aucune autre édition que celle-ci. Une manière pour l'État de contrôler la gestion des lieux de prières, en particulier les techniciens du culte tenus de réciter le Coran national. Selon Ahmed Toufik, la diffusion dans les mosquées d'une édition unique du texte sacré vise à contrer l'influence qu'entendent exercer des pays «comme l'Arabie Saoudite», précise le directeur de la fondation, en distribuant des milliers d'exemplaires du Coran fondés sur une méthode de lecture différente de celle en vigueur au Maroc : Hafs plutôt que Warsh.

Ainsi, la création d'un «Coran marocain » visant à la préservation d'un islam national met bien en évidence les enjeux contemporains que soulève la religion au Maroc où la légitimité du roi est, on l'a dit, avant tout religieuse.

Descendant de Fatima Zahra, fille du Prophète, le sultan (intronisé roi en 1955 sous le Protectorat) est le «Commandeur des croyants» (Amîr al-Mu'minîn). Par cette appellation, l'État marocain relie ensemble :

[...] deux principes d'organisation politique et religieuse qui, dans la plus grande partie du monde musulman, étaient directement antithétiques : le principe selon lequel le souverain règne parce qu'il détient une qualité surnaturelle le lui permettant; et le principe selon lequel il règne parce que les porte-parole compétents de la communauté ont collectivement reconnu qu'il devait régner. [...] Ce double fondement de la légitimité avait pour effet - peut-être serait-il plus exact de dire avait pour cause - une double perception de la nature du sultanat dans la population. D'une part, le sultan était le principal marabout du pays, le saint le plus éminent; son autorité était spirituelle. D'autre part, le sultan était le dirigeant, choisi selon les règles de la communauté islamique, son chef officiellement désigné; son autorité était politique (Geertz, $1992[1968])^{42}$.

La Commanderie des croyants (Imârat al-Mu'minîn), véritable spécificité marocaine « occupe une place centrale tant au niveau de la gestion qu’à celui de la légitimation. Elle constitue à la fois un moyen de définition des champs stratégiques réservés au roi et un mécanisme de maîtrise des rapports entre la religion et la politique» (Darif, 2010). L'islam est un élément clef de la structure de l’État.

Contrairement à d'autres pays où cohabitent plusieurs rites à la fois, la constitution marocaine confirme le référentiel islamique malékite du royaume. En vertu de son titre de «Commandeur des croyants», Mohammed VI «ne peut accepter de 
reconnaître explicitement les expressions concurrentes de l'islam. Cela équivaudrait à admettre dans la communauté un schisme qui entamerait sa vocation monopolistique et affaiblirait la légitimité chérifienne » (Tozy, 2009) - charîf désignant un descendant du prophète. C'est pourquoi, de surcroît aujourd'hui, à un moment où la monarchie est fragilisée par l'arrivée au pouvoir du PJD et la montée d'autres mouvements islamistes liés aux réseaux transnationaux d'un islam mondialisé, le roi cherche à monopoliser le champ stratégique de la religion, de manière à empêcher ces groupes et autres collectivités de faire de l'islam un référentiel propre. Dans ce but, toute autre lecture de la religion portant atteinte au rite malékite doit être écartée.

C'est pourquoi, la montée du chiisme est aussi prise au sérieux par les autorités religieuses et politiques. Selon Mohamed Sigh-Janjar³, anthropologue et directeur de la Fondation du roi Abdul-Aziz El Saoud pour les Études islamiques et les Sciences humaines à Casablanca, l'influence grandissante des communautés chiites, qui a été particulièrement visible en 2008 lors de la célébration d'Achoura ${ }^{44}$ à Tanger, a fini de convaincre le royaume de créer une édition nationale du Coran vouée à fonctionner comme une référence unique du texte de la Révélation et de celui qui s'en fait le transmetteur, Mohammed VI, dans le respect des traditions religieuses et artistiques historiquement ancrées dans le pays.

C'est donc à partir d'un mode de communication textuel conçu comme l'expression de son particularisme que le roi cherche à asseoir et consolider son autorité. À ce stade de la réflexion, de nombreuses questions subsistent néanmoins pour mieux cerner les implications religieuses et politiques de la création d'un «Coran marocain » : du point de vue des usagers et des producteurs, les spécificités du mushaf muhammadî font-elles sens? Si oui, de quelle(s) manière(s)? Derrière ces interrogations s'en profilent d'autres, plus conséquentes : dans quelle mesure la tentative de constituer une communauté soumise au roi à travers un mode de communication textuel va-t-elle réussir? Comment est-il possible d'apprécier ce succès? Dans quelques années, l'usage majoritaire (si tant est qu'il le soit) du mushaf muhammadî attestera-t-il de son hégémonie et par là de celle de la tradition malékite? Cela n'indiquera-t-il pas au contraire une crise de légitimité des élites politiques et religieuses? Autant d'interrogations auxquelles il sera possible de répondre dans une recherche ultérieure une fois que le mushaf muhammadî sera massivement distribué et utilisé par les fidèles.

43 Entretien du 9 octobre 2012 à Casablanca.

44 La fête d'Achoura correspond au dixième jour du moins de Muharram, premier mois de l'année musulmane. Elle revêt différentes significations pour le sunnisme et le chiisme : pour les uns, elle marque le début de festivités, pour les autres, c'est une journée de deuil commémorant la mort de Hussein, petit-fils du Prophète et fils d'Ali. 


\section{Conclusion}

Étudier la transformation en cours du marché éditorial coranique a montré comment l'édition du Livre saint s'articule autour de trois enjeux principaux dans le Maroc actuel : économique, religieux et politique.

Dans le cadre des pratiques éditoriales, le Coran est traité à la fois comme un objet religieux et une marchandise. Soumis à la négociation et au «piratage », il est apprécié d'un point de vue aussi bien commercial que symbolique. Le mécontentement des fidèles suscité par la circulation de corans frauduleux a mis en évidence le conflit des valeurs marchande et religieuse du Coran. Au Maroc où la religion, on l'a dit, est au cœur du processus de légitimation politique, ce conflit a conduit à une crise qui a poussé le Royaume à intervenir.

Dans le cadre des pratiques institutionnelles, le Coran représente bien plus que le texte révélé : il est aussi un objet identitaire, national et patrimonial mobilisé par le royaume pour renforcer son autorité. Dans cette perspective, deux processus ont été mis en place ${ }^{45}$. D’une part, la «nationalisation» du Coran marocain dont la publication «officielle» se veut garante de son orthodoxie, d'autre part, la recherche d'une preuve «d'authenticité » de cette édition moderne dans l'imitation des formes les plus anciennes. À cette fin, l'État a eu recours à un type de production (une forme de «lithographie moderne») qui permet de conserver l'aspect des manuscrits des origines. C'est un exemple éloquent de la manière dont, aujourd'hui au Maroc, les nouvelles technologies de l'information et de la communication reconfigurent plutôt que dissipent les structures d'autorité et de discipline qui encadrent la reproduction du texte coranique.

Étudier les processus d'édition, de marchandisation et de nationalisation du Coran a mis en évidence son caractère changeant et mobile, rompant avec l'idée communément admise qu'il est un texte sacré immuable et monolithique. Rendre visible les différentes manières dont le Coran est médiatisé par son contenant physique - le mushaf - a fait apparaître les relations de plus en plus étroites entre matérialité, religion et autorité dans le Maroc contemporain, et montré le type d'objet historique qu'il constitue pour les musulmans.

45 Enfin, lorsque le mushaf mohammadî sera massivement commercialisé dans les librairies (ceux produits jusqu’à aujourd'hui sont exclusivement destinés à être distribués gratuitement dans les mosquées), il faudra aussi interroger le Coran en tant qu’enjeu économique pour le royaume. 


\section{B IBLIOGRA PHIE}

Albin, Michael W., "Printing of the Qur'ān », Encyclopaedia of the Qur'ān. General

Editoe Dammen McAuliffe, Georgetown University, Washington DC. Brill Online, 2005, http:// referenceworks.brillonline.com/entries/encyclopaedia-of-the-quran/printing-of-the-quranCOM_OO158

AmIR-MoezzI, Mohamed (dir.), Dictionnaire du Coran, Paris, Robert Laffont, 2007.

AтіYen, George, «The Book in the Modern Arab World: The Cases of Lebanon and Egypt», dans Atiyeh George (éd.), The Book in the Islamic World. The Written Word and Communication in the Middle East, State University of New York Press, Library of Congress, 1985, p. 233-236.

AYACH, Germain, «L'apparition de l'imprimerie au Maroc », Hespéris Tamuda, 5, 1964, p. 143165.

AzAIEz, Mehdi, «Introduction», dans Azaiez et Mervin (dir.), Le Coran. Nouvelles approches, Paris, CNRS Éditions, 2013, p. 13-35.

BALAgnA, Josée, L'imprimerie arabe en Occident. XVI ${ }^{e}, X V I I^{e}$ et XVIII ${ }^{e}$ siècles, Paris, Maisonneuve \& Larose, 1984.

Benghabrit-Remaoun, Nouria, BouzoubaA, Khadija, «L'éducation préscolaire au Maroc et en Algérie », Perspectives, XXXIV, 4, 2004 (http://www.ibe.unesco.org/publications/Prospects/ProspectsPdf/132/132-f.pdf).

BlachÈre, Régis, Le Coran, Paris, PUF, 1999 [1966].

BLoom, Jonathan, Paper Before Print: The History and Impact of Paper in the Islamic World, New Haven (Connect.), Yale university Press, 2001.

Bourqia, R., El AyAdi, M., El HARrAs, R., RACHIK, H., Les jeunes et les valeurs religieuses, Casablanca, Eddif, 2000.

BrocketT, Alan, Studies in Two Transmissions of the Qur'an, thèse disponible en ligne : http:// fr.scribd.com/doc/21972348/Studies-in-Two-Transmissions-of-the-Qur-an-by-Adrian-AlanBrockett, 1984.

Burton, Jean, Encyclopédie de l'islam, tome VII, Paris, Maisonneuve \& Larose, 1993 [1966].

CHARTIER, Roger, Lectures et lecteurs dans la France d'Ancien Régime, Paris, Seuil (L'Univers historique), 1987.

CHARTIER, Roger, Culture écrite et société. L'ordre des livres (XVI'-XVIII ${ }^{e}$ siècle), Paris, Albin Michel, 1996.

CHARTIER, Roger, MARTIN Henri-Jean, Histoire de l'édition française (4 vol.), Paris, Fayard, 1989-1990 [1986-1990].

CoHen, Anouk, «Le Coran et ses multiples formes », Terrain, 59, 2012, p. 7 0-87.

CoHEN, Anouk, «Le livre du Coran à Casablanca et à Rabat», Archives des sciences sociales des religions, 150, 2010, p. 175-195.

CoLE, Juan, «Printing and Urban Islam in the Mediterranean World, 1890-1920 ", dans Leila Tarazi Fawaz et C. A. Baily (éd.), Modernity and Culture. From the Mediterranean to the Indian Ocean, New York, Columbia University Press, 2002, p. 344-364.

DARIF, Mohamed, Monarchie marocaine et acteurs religieux, Casablanca, Afrique Orient, 2010.

DE BLIC, Véronique, Le marché de l'édition au Maroc, Rabat, Direction des relations économiques extérieures, Poste d'expansion économique, 2000.

DE PrÉMARE, Alfred-Louis, Aux origines du Coran. Questions d'hier, approches d'aujourd'hui, Paris, Téraèdre, 2004. 
DÉRoche, François, Le livre manuscrit arabe, Paris, BNF, 2005.

DÉroche, François, Le Coran, Paris, PUF (Que sais-je), 2008 [2005].

Eickelman, Dale, Knowledge and Power in Morocco, Princeton (NJ), Princeton, 1992.

EISEnsteIn, Elizabeth, The Printing Press as an Agent of Change. Communication and Cultural Transformations in Early Modern Europe, Cambridge, Cambridge University Press, 1979.

El AYADI, Mohammed, RACHIK Hassan, TozY Mohammed, L'islam au quotidien. Enquête sur les pratiques religieuses au Maroc, Casablanca, Prologue, 2007.

El YazAmi, Abdelali, La lecture au Maroc, Casablanca, El Maârif, 2007.

EMPERADOR, Montserrat, «Diplômés chômeurs au Maroc : dynamiques de pérennisation d'une action collective plurielle », L'Année du Maghreb, 2007, p. 297-311.

FortiER, Corinne, «"Une pédagogie coranique”. Modes de transmission des savoirs islamiques (Mauritanie) », Cahiers d'études africaines, XLIII(1-2), 169-170, 2003, p. 235-26o.

GDouRA, Wahid, Le début de l'imprimerie arabe à Istanbul et en Syrie : évolution de l'environnement culturel (1706-1787), Tunis, Publications de l'Institut supérieur de documentation, 1985.

GeERTz, Clifford, Observer l'islam, Paris, La Découverte, 1968 [1992].

GEERTZ, Clifford, Savoir local, savoir global. Les lieux du savoir, Paris, PUF, 1986.

GraHAM, William A., Beyond the Written Word: Oral Aspects of Scripture in the History of Religion, Cambridge, Cambridge University Press, 1987.

HAMÈs, Constant, «Coran et traditions prophétiques, droit musulman, savoirs talismaniques », dans Aurélie Bosc et Mireille Jacotin (dir.), Le goût de l'Orient, Milan, Silvana Editoriale, 2013.

HAMÈs, Constant (dir.), Coran et talismans, Paris, Karthala, 2007.

Hammoud, Abdellah, Une saison à La Mecque. Récit de pèlerinage, Paris, Seuil, 2005.

HiRschKInd, Charles, "Media and the Qur'ān », Encyclopaedia of the Qur'ān. General Editor : Jane Dammen McAuliffe, Georgetown University, Washington DC. Brill Online, 2005 (http:// referenceworks.brillonline.com/entries/encyclopaedia-of-the-quran/media-and-the-quranCOM_oo117).

HouARI, Touati, Islam et voyage au Moyen-Âge. Histoire et anthropologie d'une pratique lettrée, Paris, Seuil, 2000.

LAPLANCHE, François, La Bible en France. Entre mythe et critique. XVI $I^{e} X I X^{e}$ siècle, Paris, Albin Michel, 1994.

LARCHER, Pierre, «Le Coran : l'écrit, le lu, le récité », dans Mehdi Azaiez (dir.), Le Coran. Nouvelles approches, Paris, CNRS Éditions, 2013, p. 243-256.

LAROUI, Abdellah, Les origines sociales et culturelles du nationalisme marocain, 1830-1912, Casablanca, Centre culturel arabe, 1993.

LASSAVE, Pierre, L'appel du texte. Sociologie du savoir bibliste, Rennes, Presses universitaires de Rennes, 2011.

Macdonald, D. B. , Encyclopédie de l'islam, tome III, Paris, Maisonneuve \& Larose, 1971 [1960].

MCKenzIE, Donald, La bibliographie et la sociologie des textes, Paris, Éditions du cercle de la librairie, 1991 [1986].

MERMIER, Franck, Le livre et la ville. Beyrouth et l'édition arabe, Arles, Actes Sud/Sindbad, 2005 .

NASRALlaH, Joseph, L'imprimerie au Liban, Beyrouth, Bibliographie de l'université Saint-Jospeh, 1951.

Pedersen, Johannes, The Arabic Book, Princeton, Princeton University Press, 1984. 
Pouessel, Stéphanie, «Écrire la langue berbère au Royaume de Mohammed VI : Les enjeux politiques et identitaires du tifinagh au Maroc », Revue des Mondes musulmans et de la Méditerranée, 124, 2008, p. 101-128.

REZVAN, Efim, «Orthography», Encyclopaedia of the Qur'ān. General Editor: Jane Dammen McAuliffe, Georgetown University, Washington DC, 2005.

Robinson, Francis, «Technology and Religious Change: Islam and the Impact of Print», Modern Asian Studies, 27/1, 1993, p. 229-251.

RoBinson, Francis, «Islam and the Impact of Print in South Asia », dans Robinson Francis (éd.), Islam and Muslim History in South Asia, Delhi, Oxford University Press, 200o, p. 66-104.

RoBson J. , «Isn'ād. » Encyclopédie de l'Islam, Brill Online, 2005, http://referenceworks.brillonline.com/entries/encyclopedie-de-l-islam/isnad-SIM_3665

SAntini, Maud, Paris, librairie arabe, Marseille/Aix-en-Provence, Parenthèses/MMSH, 2006.

SGHIR Janjar, Mohammed, «L'édition dans le Maroc indépendant : 1955-2003», Rapport thématique : Dimensions artistiques, culturelles et spirituelles, 50 ans de développement humain. Perspectives 2025, Casablanca, 2006, p. 43-61.

StARRETT, Gregory, Putting Islam to Work: Education, Politics, and Religious Transformation in Egypt, Berkeley, University of California Press, 1998.

STARRETT, Gregory, «The Political Economy of Religious Commodities in Cairo », American Anthropologist, 97/1, 1995, p. 51-68.

Tozy, Mohamed, Monarchie et islam politique au Maroc, Paris, Presses de Science Po, 1999.

Tozy, Mohamed, «L'évolution du champ religieux marocain au défi de la mondialisation », Revue internationale de politique comparée, 16/1, 2009, p. 63-81.

UnESCO, Production de livres et lecture dans le monde arabe, 1987.

UnESCO, Literacy for Life. Des options réelles pour les politiques et les pratiques - Le Maroc, 2005 .

Vermeren, Pierre, Le Maroc en transition, Paris, La Découverte, 2001.

ZEGHAL, Malika, Les islamistes marocains. Le défi à la monarchie, Paris, La Découverte, 2005. 